(1)

CrossMark

\title{
Triple therapy trials in COPD: a precision medicine opportunity
}

\author{
Samy Suissa ${ }^{1}$ and Amnon Ariel $^{2}$
}

Affiliations: ${ }^{1}$ Center for Clinical Epidemiology, Lady Davis Institute, Jewish General Hospital, and the Depts of Epidemiology and Biostatistics and of Medicine, McGill University, Montreal, QC, Canada. ${ }^{2}$ Lung Unit, Emek Medical Center, Clalit Healthcare Services, Afula, Israel.

Correspondence: Samy Suissa, Centre for Clinical Epidemiology, Jewish General Hospital, 3755 Cote Ste-Catherine, Montreal, QC, Canada H3T 1E2. E-mail: samy.suissalamcgill.ca

@ERSpublications

Recent trials comparing triple therapy with LAMA/LABA in COPD reveal a peculiar pattern in the timing of exacerbations, with the benefit of triple therapy exclusively in the first month, suggesting a precision medicine opportunity in COPD treatment http://ow.ly/WBFb30m9ZeM

Cite this article as: Suissa S, Ariel A. Triple therapy trials in COPD: a precision medicine opportunity. Eur Respir J 2018; 52: 1801848 [https://doi.org/10.1183/13993003.01848-2018].

\section{Introduction}

The Global Initiative for Chronic Obstructive Lung Disease (GOLD) strategy document for the treatment of chronic obstructive pulmonary disease (COPD) suggests that, after bronchodilator treatment with both long-acting muscarinic antagonists and long-acting inhaled $\beta$-agonists (LAMA/LABA), the addition of inhaled corticosteroids (ICS) should be reserved for patients with frequent moderate or severe COPD exacerbations [1]. The GOLD group emphasises the scarcity of evidence and the need for systematic research on this escalation from dual bronchodilator to triple therapy (LAMA/LABA/ICS) [2].

The recent TRIBUTE and IMPACT trials assessed such escalation using a LAMA/LABA as comparator $[3,4]$. The design of the IMPACT trial raised some methodological issues that limited the interpretation of its findings [5]. However, these limitations provide some opportunities to better understand the potential for triple therapy to advance precision medicine, which has been lagging behind in COPD $[6,7]$.

\section{The TRIBUTE and IMPACT trials}

The TRIBUTE trial compared single-inhaler triple therapy (glycopyrronium bromide, formoterol fumarate and beclomethasone dipropionate), administered twice daily, versus a dual LAMA/LABA bronchodilator combination (glycopyrronium plus indacaterol) on the rate of COPD exacerbations over 52 weeks [3]. The study included 1532 COPD patients with severe to very severe airflow limitation (GOLD grade 3-4) and at least one moderate to severe exacerbation in the previous year. While patients with current asthma were excluded, those with a past "asthma diagnosis" could participate. All patients discontinued their maintenance therapy and switched to the LAMA/LABA comparator during a 2-week run-in before randomisation. Triple therapy lowered the rate of moderate-to-severe exacerbations by $15 \%$ compared with the LAMA/LABA comparator.

The IMPACT trial compared single-inhaler triple therapy (umeclidinium, vilanterol and fluticasone furoate) with its two dual-drug inhaler comparators, LABA/ICS (vilanterol and fluticasone furoate) and LAMA/ LABA (umeclidinium and vilanterol) over 52 weeks [4]. This trial recruited 10355 COPD patients with moderate-to-severe airflow limitation (GOLD grade 2-4) and a recent history of exacerbations. Patients with current asthma were excluded, but those with a "past diagnosis" of asthma participated. Patients continued 
their pre-study treatment during a 2-week run-in before randomisation. Triple therapy lowered the rate of moderate-to-severe exacerbations by $25 \%$ compared with LAMA/LABA and by $15 \%$ compared with LABA/ ICS. Mortality, a secondary end-point, was lower with triple therapy by $42 \%$ compared with LAMA/LABA.

\section{Timing of exacerbations in TRIBUTE and IMPACT}

TRIBUTE and IMPACT displayed the cumulative incidence curves of a moderate or severe exacerbation comparing triple therapy with LAMA/LABA (figure $1 \mathrm{~b}$ from IMPACT [4] and supplementary figure 3 from TRIBUTE [3]). To provide a more telling perspective on the pattern of exacerbation over time, we converted the cumulative incidence curves into monthly rates of exacerbation. Because we did not have access to the data, we digitised the figures to obtain the values at each point of the cumulative incidence curves. The rate, derived from the average slope of the cumulative incidence curve, was approximated for each month of follow-up by the difference between the digitised cumulative incidence value at the end of each month with that at the beginning of that month [8].

Figure $1 \mathrm{a}$ and $\mathrm{b}$ show the resulting approximated rates of a first moderate or severe exacerbation for each of the 12 months of follow-up, by treatment group, for the IMPACT and TRIBUTE trials, respectively, joined by smoothing. The figures clearly show that the difference in the rate of exacerbation between LAMA/LABA/ICS and LAMA/LABA over follow-up is due to the first-month's surge, with practically no differences in the subsequent rates between the two groups. This transient effect of ICS discontinuation is especially prominent in IMPACT where, in the first month, the incidence rate of first exacerbation under LAMA/LABA is 15.0 per 100 per month compared with 8.7 under LAMA/LABA/ICS (figure 1a). By contrast, the mean incidence rate over the remaining 11 months under LAMA/LABA is 3.4 per 100 per month compared with 3.7 under LAMA/LABA/ICS. Therefore, triple therapy is associated with a decrease of 6.3 per 100 per month in the incidence of exacerbation during the first month of treatment and a trivial increase of 0.3 per 100 per month over each of the remaining 11 months.

Similarly, for TRIBUTE, the first month incidence rates of 2.8 versus 7.1 per 100 per month, respectively for triple therapy and LAMA/LABA, led to a decrease in the incidence of exacerbation with triple therapy in the first month of 4.3 per 100 per month (figure 1b). Over the remaining 11 months, the mean incidence rates of 2.9 and 2.7 per 100 per month, respectively for triple therapy and LAMA/LABA, led to a negligible increase of 0.2 per 100 per month.

These calculations were repeated for the reported $42 \%$ lower mortality with triple therapy when compared with LAMA/LABA in the IMPACT trial [9]. Figure 2a displays the rate of death per month over time for the IMPACT trial, approximated from the mortality curves [9]. Here again, the difference in mortality between LAMA/LABA/ICS (rate 0.10 per 100 per month) and LAMA/LABA (rate 0.30 per 100 per month) is localised in the first 4 months of follow-up. In the subsequent 8 months of follow-up, mortality is 0.12 per 100 per month, equally under LAMA/LABA/ICS and LAMA/LABA.

\section{Data interpretation}

The early higher incidence of exacerbation with LAMA/LABA, compared with triple therapy, and followed by no subsequent differences as observed in these trials (figures 1 and 2a), a manifestation known as
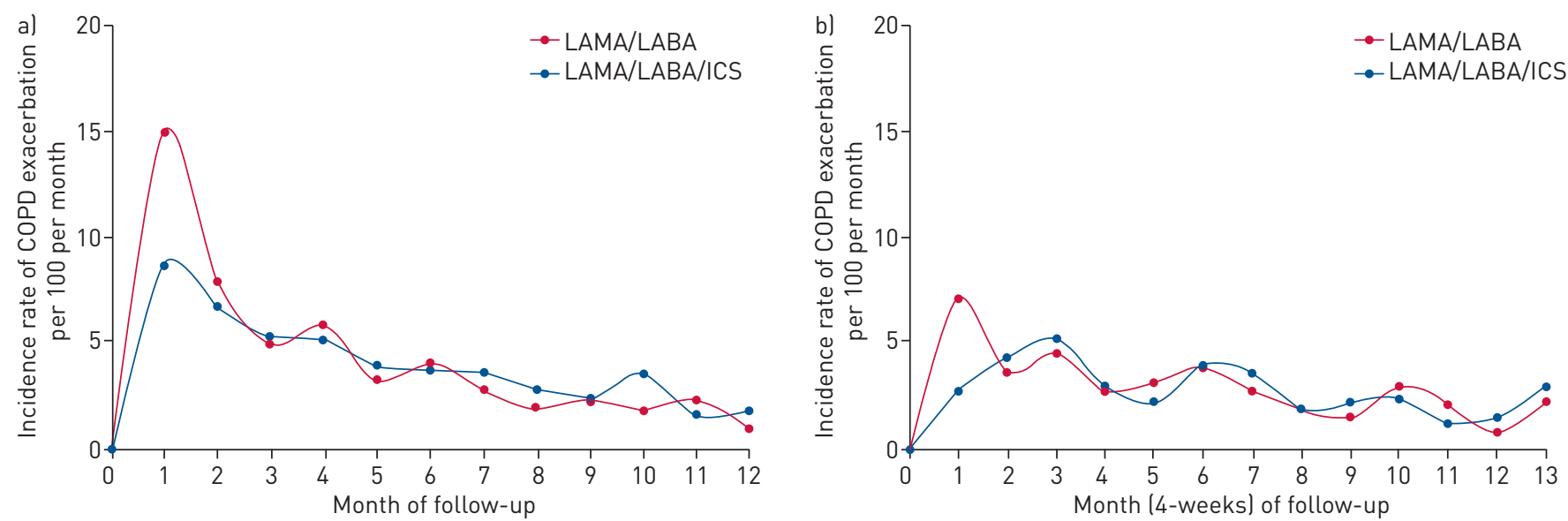

FIGURE 1 Monthly rate (per 100 per month) of a first moderate or severe exacerbation under long-acting muscarinic antagonists (LAMA)/

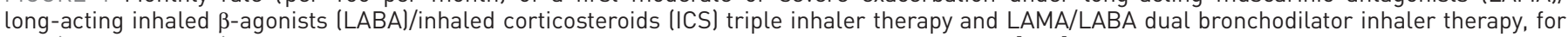
the a) IMPACT and b) TRIBUTE trials, approximated from the time-to-first exacerbation curves [3, 4]. 

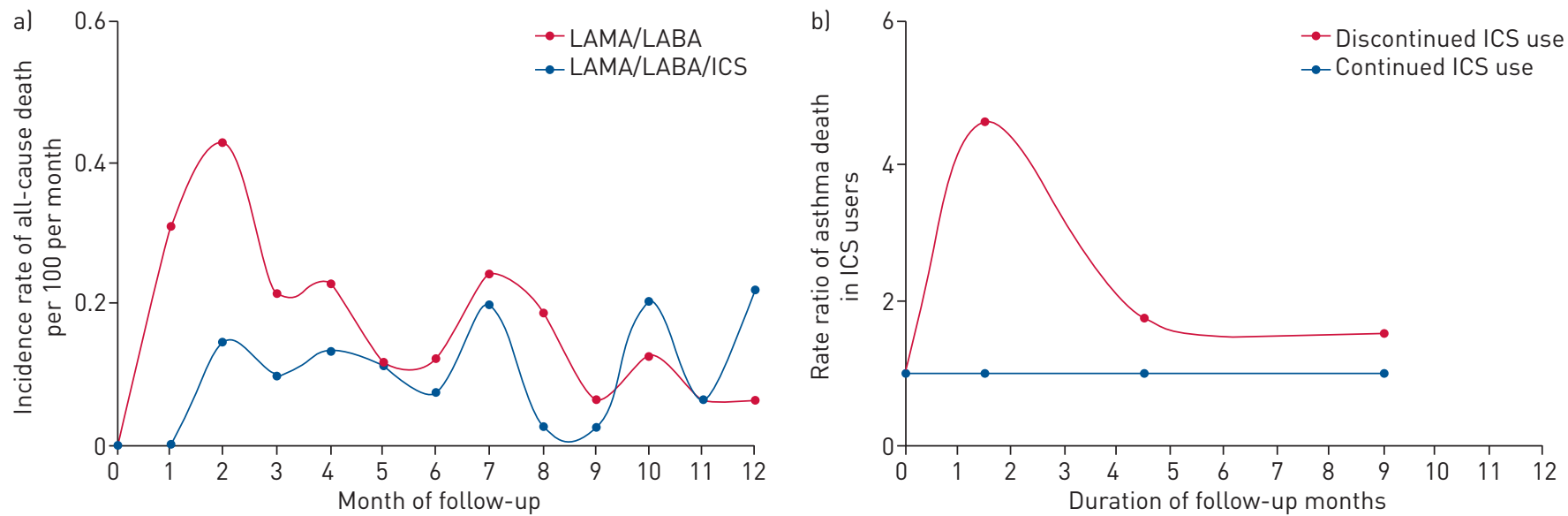

FIGURE 2 a) Monthly rate of death (per 100 per month) under long-acting muscarinic antagonists (LAMA)/long-acting inhaled $\beta$-agonists (LABA)/ inhaled corticosteroids (ICS) triple inhaler therapy and LAMA/LABA dual bronchodilator inhaler therapy in the IMPACT trial, approximated from the survival curves [9]. b) Rate ratio of asthma death after ICS discontinuation, compared with ICS continuation, from a cohort study of 30569 patients with asthma on ICS effectiveness in lowering asthma mortality [16].

"depletion of susceptibles", can be informative to understand a treatment's effect, including in randomised controlled trials [10-12]. Indeed, such an observation suggests that there is a subset of patients who could benefit from triple therapy, while the others benefit at least equally from LAMA/LABA. In identifying potential effect-modifying factors that can help to characterise this subset of responsive patients, two are rather conspicuous, namely study population selection and the treatment withdrawal at randomisation.

The TRIBUTE study population had a mean forced expiratory volume in $1 \mathrm{~s}$ (FEV1) of $36 \%$ predicted and $20 \%$ had two or more exacerbations in the prior year. By contrast, the IMPACT study population, which selectively excluded patients with $\mathrm{FEV}_{1}>50 \%$ predicted if they had less than two exacerbations in the previous year, resulted in a population with a mean FEV1 of $45 \%$ predicted, pronounced bronchodilator reversibility (18\% versus $8.6 \%$ in TRIBUTE), and $54 \%$ with two or more exacerbations in the previous year, which is exceedingly disproportionate compared with typical COPD populations [13]. Moreover, both TRIBUTE and IMPACT allowed the inclusion of patients with a "history of asthma", a significant risk factor for severe COPD exacerbations [14].

In TRIBUTE and IMPACT, maintenance treatment was withdrawn at the time of randomisation, particularly prior ICS use. While this will not affect patients randomised to ICS-containing therapy, it will affect the LAMA/LABA comparator arm. The potential effect is considerable since around $65 \%$ and $70 \%$ of patients were pre-study users of ICS in TRIBUTE and IMPACT, respectively.

These two factors together, namely the inclusion of patients with past asthma and the withdrawal of ICS in patients for whom ICS are indicated, could potentially induce an early surge in exacerbations in the LAMA/ LABA patients, possibly amplified by the selective inclusion in IMPACT of GOLD group 2D patients, who contributed around a third of the study population while disproportionately accounting for two-thirds of the group D frequent exacerbators. In addition, the possible adrenal insufficiency from ICS therapy and potential adrenal rebound effect from its withdrawal could have contributed to the effects [15].

These effects could also explain the IMPACT trial's findings on mortality, which may be the result of an excess mortality in the LAMA/LABA arm [9]. Indeed, discontinuing ICS treatment in patients with asthma has been shown to increase asthma mortality in a cohort study of over 30000 patients with asthma [16]. Figure $2 \mathrm{~b}$ depicts the increase in the rate ratio of asthma death, as a function of time since ICS discontinuation, particularly evident in the first 3 months after discontinuation where the risk of death is increased over four-fold [16]. This early mortality pattern matches the one from the IMPACT trial (figure 2a).

\section{Conclusion}

The TRIBUTE and IMPACT trials reported that triple therapy, compared with a LAMA/LABA bronchodilator, lowered the incidence of COPD exacerbations over a 1-year follow-up. We demonstrated that the timing of the exacerbations in these trials presents a particularly informative pattern. It shows that the lower rate of a first exacerbation with triple therapy is exclusively due to a lower rate in the first month of follow-up, while the rate was comparable to LAMA/LABA in the subsequent 11 months. This pattern of "depletion of susceptibles" suggests that there is a subset of patients who could benefit from triple therapy, while the remaining patients benefit equally from LAMA/LABA. 
We submit that the history of asthma and the prior use of ICS, withdrawn at randomisation, could be two important factors of interest in identifying such subsets of responders. Another could be the unusually overrepresented patients in IMPACT with $\mathrm{FEV}_{1}>50 \%$ predicted and two or more exacerbations in the previous year. The data could be reanalysed to explore these factors and others, including thresholds of airflow limitation and eosinophil counts, particularly higher cut-offs such as $4 \%$ or 300 cells $\mu \mathrm{L}^{-1}[7,17$ 19]. It would also be valuable to see whether the pattern over time also emerges with the frequency of exacerbations and to compare the characteristics of early and late exacerbators.

The inclusion of patients with a history of asthma may explain the difference in findings between the IMPACT and the FLAME trial, in comparing LAMA/LABA with LABA/ICS dual bronchodilators [20]. IMPACT found a lower rate of exacerbations with LABA/ICS than with LAMA/LABA (1.07 versus 1.21, respectively), opposite to that of the FLAME trial (1.19 versus 0.98). An important difference between the trials is the inclusion of patients with a past asthma diagnosis, excluded in the FLAME trial, as well as the approach to the run-in period $[20,21]$.

Future trials on the escalation from dual bronchodilator to triple therapy in COPD could strive to identify the properly targeted study populations, for example avoiding patients with a history of asthma, and to carefully address withdrawal of ICS at the time of randomisation. In the case of broadly selected study populations, pre-specified stratification of the results by important effect-modifiers, such as prior asthma, airflow limitation, exacerbation frequency and the degree of eosinophilia, could provide a precision medicine approach to COPD management. Such a modern approach will permit the identification of subsets of patients who could benefit from triple therapy and avoid harms in a number of patients for whom triple therapy is not more effective than dual bronchodilators.

Conflict of interest: S. Suissa reports personal fees from AstraZeneca (payment for lectures including service on speakers' bureaus), grants from Boehringer Ingelheim (research grants and participated in advisory board meetings) and grants from Novartis (research grants and participated in advisory board meetings), outside the submitted work. A. Ariel reports personal fees from AstraZeneca, GlaxoSmithKline and Kamada, and personal fees and non-financial support from Boehringer Ingelheim, outside the submitted work.

\section{References}

1 Vogelmeier CF, Criner GJ, Martinez FJ, et al. Global Strategy for the Diagnosis, Management, and Prevention of Chronic Obstructive Lung Disease 2017 Report: GOLD Executive Summary. Eur Respir J 2017; 49: 1700214.

2 Rodriguez-Roisin R, Rabe KF, Vestbo J, et al. Global Initiative for Chronic Obstructive Lung Disease (GOLD) 20th Anniversary: a brief history of time. Eur Respir J 2017; 50: 1700671.

3 Papi A, Vestbo J, Fabbri L, et al. Extrafine inhaled triple therapy versus dual bronchodilator therapy in chronic obstructive pulmonary disease (TRIBUTE): a double-blind, parallel group, randomised controlled trial. Lancet 2018; 391: 1076-1084.

4 Lipson DA, Barnhart F, Brealey N, et al. Once-daily single-inhaler triple versus dual therapy in patients with COPD. N Engl J Med 2018; 378: 1671-1680.

5 Suissa S, Drazen JM. Making sense of triple inhaled therapy for COPD. N Engl J Med 2018; 378: 1723-1724. Collins FS, Varmus H. A new initiative on precision medicine. N Engl J Med 2015; 372: 793-795.

7 Sidhaye VK, Nishida K, Martinez FJ. Precision medicine in COPD: where are we and where do we need to go? Eur Respir Rev 2018; 27: 180022.

8 Kalbfleisch JD, Prentice RL. The Statistical Analysis of Failure Time Data. New York, John Wiley \& Sons, Ltd, 1980.

9 Lipson DA BF, Brealey N, Day NC, et al. Reduction in all-cause mortality with single inhaler triple therapy (FF/ UMEC/VI) versus dual therapy (FF/VI and UMEC/VI) in symptomatic patients with COPD: prespecified analysis of the phase III IMPACT trial. Am J Respir Crit Care Med 2018; 197: A1015.

10 Miettinen OS, Caro JJ. Principles of nonexperimental assessment of excess risk, with special reference to adverse drug reactions. J Clin Epidemiol 1989; 42: 325-331.

11 Renoux C, Dell'Aniello S, Brenner B, et al. Bias from depletion of susceptibles: the example of hormone replacement therapy and the risk of venous thromboembolism. Pharmacoepidemiol Drug Saf 2017; 26: 554-560.

12 Hernán MA. The hazards of hazard ratios. Epidemiology 2010; 21: 13-15.

13 Hurst JR, Vestbo J, Anzueto A, et al. Susceptibility to exacerbation in chronic obstructive pulmonary disease. N Engl J Med 2010; 363: 1128-1138.

14 Müllerova H, Maselli DJ, Locantore N, et al. Hospitalized exacerbations of COPD: risk factors and outcomes in the ECLIPSE cohort. Chest 2015; 147: 999-1007.

15 Lapi F, Kezouh A, Suissa S, et al. The use of inhaled corticosteroids and the risk of adrenal insufficiency. Eur Respir J 2013; 42: 79-86.

16 Suissa S, Ernst P, Benayoun B, et al. Low-dose inhaled corticosteroids and the prevention of death from asthma. N Engl J Med 2000; 343: 332-336.

17 Bafadhel M, Pavord ID, Russell REK. Eosinophils in COPD: just another biomarker? Lancet Respir Med 2017; 5: 747-759.

18 Suissa S, Ernst P. Precision medicine urgency: The case of inhaled corticosteroids in COPD. Chest 2017; 152: 227-231.

19 Suissa S, Dell'Aniello S, Ernst P. Comparative effectiveness of LABA-ICS versus LAMA as initial treatment in COPD targeted by blood eosinophils: a population-based cohort study. Lancet Respir Med 2018; 6: 855-862.

20 Wedzicha JA, Banerji D, Chapman KR, et al. Indacaterol-glycopyrronium versus salmeterol-fluticasone for COPD. N Engl J Med 2016; 374: 2222-2234.

21 Suissa S. Run-in bias in randomised trials: the case of COPD medications. Eur Respir J 2017; 49: 1700361. 